\title{
Candidiasis hiperplásica crónica de la lengua: Una lesión con cambios displásicos
}

\author{
Chronic hyperplastic candidiasis of the tongue: A lesion with dysplastic changes
}

\author{
Wilson Delgado Azañero ${ }^{1, a}$, Manuel Arrascue Dulanto ${ }^{1, b}$, Sandro Lévano Loayza ${ }^{1, c}$
}

\section{RESUMEN}

La candidiasis hiperplásica crónica (CHC), es una infección micótica oral producida por la especie C. albicans, que se presenta en personas inmunocompetentes como una placa blanca que no desaparece al raspado y que puede ser confundida con la leucoplasia, entidad relacionada con el carcinoma oral. Para su diagnóstico es indispensable la toma de una biopsia que permita identificar la presencia de hifas y blastosporas de candida invadiendo el epitelio. En este trabajo se analizan las características clínicas y la probable causa de una CHC del dorso de la lengua diagnosticada en un paciente masculino de 54 años. Se presenta y discute la distribución del hongo dentro del epitelio, así como las alteraciones inducidas en el fenómeno de proliferación y diferenciación celular de este tejido. El tratamiento se fundamentó en la corrección del factor etiológico identificado, la eliminación mecánica de la placa micótica y la prescripción de medicación antifúngica. Debido a sus características clínicas la CHC se puede confundir con la lesión potencialmente maligna oral denominada leucoplasia. La biopsia de la lesión es fundamental para establecer el diagnóstico el cual conducirá a evitar sobre tratamientos.

PALABRAS CLAVE: Candidiasis hiperplásica, Candida albicans, Candidiasis oral.

\begin{abstract}
\footnotetext{
Facultad de Estomatología Roberto Beltrán, Universidad Peruana Cayetano Heredia. Lima, Perú.

Profesor Emérito, Especialista en Medicina y Patología Oral y Maxilofacial.

Profesor Asociado de Cirugía Oral y Maxilofacial. Mg Esp Cirugía Bucal y Maxilofacial

Cirujano Dentista, Diplomado en Cirugía Oral
}

Chronic hyperplastic candidiasis (CHC) is an oral fungal infection produced by $C$. albicans specie. It occurs in the oral mucosa of immunocompetent persons and presents as a white plaque that cannot be detached upon rasping. Due to this feature, can be confused with leukoplakia, a potentially malignant lesion related to the development of squamous cell carcinoma. The diagnosis is established by biopsy that allows identifying hifas and blastospores of candida invading the epithelium and inducing alterations in the cell proliferation and differentiation of this tissue. The clinical features and probable etiology agent of a case of CHC located in the dorsum of the tongue diagnosed in a 54-years- old male are presented. Histological demonstration of the fungus in the different layers of the epithelium and the alterations induced in the cell proliferation and differentiation of 
this tissue are discussed. Treatment was based on the correction of the etiologic factor, mechanical elimination of the mycotic plaque and use of antifungal medication. Due to their clinical characteristics, CHC can be confused with the oral potentially malignant lesion called leukoplakia. Biopsy of the lesion is absolutely necessary to establish the diagnosis, to identify dysplastic changes and therefore avoid over treatment.

KEYWORDS: Hyperplastic candidiasis; Candida albicans; oral candidiasis.

\section{INTRODUCCIÓN}

Candidiasis o candidosis son términos sinónimos que se usan para referirse a una infección relativamente frecuente de la cavidad oral producida por el hongo de la especie Candida albicans. El sufijo iasis generalmente se usa para las infecciones por parásitos como por ejemplo teniasis y la terminación osis para referir a infecciones por hongos, ejemplo histoplasmosis. En Estomatología es más común usar el término candidiasis para la infección que es motivo de este artículo. El término moniliasis usado antiguamente no es correcto ya que la monilia o monilinea es un género de hongos de la familia Sclerotiniaceae que produce enfermedad en los árboles frutales como el cacao (1).

La candidiasis oral es producida principalmente por la especie Candida albicans, pero también se han reportado casos producidos por las especies $C$. glabrata, C. guillermondii, C.kruesi, C.lusitaniae, C.parapsilosis, C.pseudotropicalis, C. stellatoidea, y C. tropicalis (2).

La mayoría de personas albergan en su boca géneros de candida, habiéndose reconocido que del 30 al $60 \%$ son portadores sanos. Este microorganismo se considera un comensal más que un patógeno de la cavidad oral (2). La mayoría de estudios epidemiológicos sobre candida se han realizado cultivando el hongo en individuos que no tenían signos ni sintomatología de infección, de tal manera que, si se utiliza el cultivo como procedimiento de diagnóstico de la enfermedad, se obtendrán muchos falsos positivos, en consecuencia, es muy importante diferenciar entre la presencia de candida como comensal y candida como patógeno $(2,3)$.

La transformación de un organismo comensal a patógeno depende de la presencia de factores que modifiquen el medio ambiente favoreciendo el desarrollo de la infección oportunista (3). Para candidiasis los factores implicados son: 1. Presencia de irritantes crónicos locales. 2. Falta de higiene de aparatos protésicos. 3. Aplicación tópica, en aerosol o ingesta de corticoesteroides. 4. Xerostomía. 5. Factores dietéticos. 6. Desórdenes inmunológicos. 7. Desórdenes endocrinos. 8. Enfermedades crónicas malignas y no malignas. 9. Discrasias sanguíneas severas. 10. Radiación de la cabeza y el cuello. 11. Nutrición deficiente. 12. Edad avanzada. 13. Hábito de fumar. 14. Displasia epitelial (3-5).

La C. albicans coloniza la superficie de la mucosa oral y causa daño debido a las siguientes características: adherencia a las células huésped, hidrofobicidad y secreción de enzimas hidrolíticas como: lipasas, fosfolipasas y proteinasas. Su virulencia radica en su capacidad de adaptarse a diferentes hábitats y a su integración a comunidades microbianas que se adhieren a la superficie de los tejidos conocidas como biopelículas (5).

La infección por candida produce varios cuadros clínicos. Holmstrup y Axel en el año 1990 (6) propusieron la siguiente clasificación: 1. Candidiasis pseudomembranosa aguda y crónica. 2. Candidiasis eritematosa aguda y crónica 3. Candidiasis hiperplásica crónica y 4. Candidiasis asociada a otros factores: estomatitis protésica, queilitis angular y glositis romboidal media. Posteriormente se agregó al cuarto grupo el eritema gingival lineal asociado a infección por VIH.

Los cuadros mencionados corresponden a candidiasis primaria de la boca, en cambio se denomina candidiasis oral secundaria cuando está presente en entidades sistémicas que cursan con candidiasis mucocutánea, afectación de las uñas, de la región vulva vaginal y ocasionalmente con compromiso de la laringe, faringe y esófago. Ejemplo de estas entidades son la candidiasis mucocutánea 
crónica familiar, el síndrome de endocrinopatía con candidiasis, el síndrome de Di George, etc. (7).

La candidiasis pseudomembranosa se caracteriza por la aparición de placas blancas sobre la superficie de la mucosa oral que se desprenden al rasparlas con un instrumento de bordes romos dejando un área eritematosa. Mayormente afecta la lengua, paladar blando y carrillos, en algunos casos toda la boca puede estar comprometida. Este tipo de candidiasis está asociado al uso de corticoides tópicos o en aerosoles, ocurre con alta frecuencia en personas con inmunosupresión por VIH o a otro tipo de inmunosupresión (8).

La candidiasis eritematosa se manifiesta como un enrojecimiento localizado en la mucosa con o sin síntomas, afecta principalmente el dorso de la lengua y el paladar duro, está asociado con el uso de antibióticos de amplio espectro, corticoesteroides e inmunosupresión por el VIH. En el dorso de la lengua se presenta como una zona depapilada de color rojo. La demostración del hongo se hace mediante frotices tomados de las áreas eritematosas y teñidos con PAS $(8-10)$.

La candidiasis asociada a uso de dentaduras se denomina estomatitis subplaca, se presenta como un área eritematosa puntiforme, lisa o granular, restringida a la zona de la mucosa del paladar cubierta por una dentadura de acrílico, con frecuencia va acompañada de queilitis angular y glositis romboidal media $(8,9)$.

La queilitis angular por candida puede estar relacionada con pérdida de la dimensión vertical en portadores y no portadores de prótesis o asociada a la deficiencia de vitamina B12 y anemias ferropénicas. Es importante señalar que algunos casos de queilitis angular pueden estar asociados a infección por staphylococcus y streptococcus y no a candida (10). La glositis romboidal media asociada a candidiasis se presenta como una mácula eritematosa con forma de rombo o elíptica ubicada en la parte media del dorso de la lengua inmediatamente delante de las papilas caliciformes. Histológicamente se observa ausencia de papilas filiformes, epitelio con crestas epiteliales hiperplásicas y presencia de hifas de candida en la superficie del epitelio $(9,10)$.
El eritema gingival lineal es una forma de candidiasis que ocurre mayormente en pacientes VIH positivos, originalmente fue denominado gingivitis marginal por VIH. Se presenta como una banda eritematosa de 2 a $3 \mathrm{~mm}$ de ancho ubicada en la encía marginal que se continúa con un eritema difuso en la encía adherida. En estos casos se ha identificado además de C. albicans a la C. dubliniensis (8).

La candidiasis hiperplásica crónica ( $\mathrm{CHC})$ denominada también leucoplasia por candida es una forma de candidiasis oral de aparición infrecuente que se puede confundir con la lesión potencialmente maligna denominada leucoplasia. Se presenta como placa blanquecina elevada, palpable, bien demarcada, que no desaparecen al raspado. Debido a estas características se puede confundir con la leucoplasia homogénea y cuando además del color blanco presenta pequeñas áreas eritematosas se asemeja a la leucoplasia moteada. Una característica relevante es que no desaparece con el raspado (11).

Generalmente se presenta en los carrillos, bordes de la lengua o en forma bilateral en las comisuras labiales, el paladar es la zona menos afectada. En este tipo de candidiasis la especie más frecuentemente encontrada es la $C$. albicans (11-15). Como agentes contribuyentes para el desarrollo de $\mathrm{CHC}$ se ha sugerido al trauma y a la fricción crónica por dientes en mala posición o prótesis dentales mal confeccionadas o deterioradas que al actuar sobre la mucosa oral producen áreas ásperas que constituyen sitios propicios para la adherencia y desarrollo de la candida (15).

También se ha propuesto que el hábito de fumar está directamente ligado al desarrollo de $\mathrm{CHC}$ debido a que el tabaco induce queratinización epitelial, reduce los niveles de inmunoglobulinas A en saliva y posiblemente deprime la función del leucocito polimorfonuclear (11), célula que tiene la capacidad de fagocitar a especies de candida para mantener el equilibrio de la flora oral.

En la CHC las hifas del hongo no sólo se encuentran en la superficie del epitelio, sino que invaden los estratos subyacentes induciendo cambios displásicos con el riesgo de transformación maligna $(9,11-15)$. Si bien la identificación del hongo se puede realizar mediante examen directo de frotices teñidos con 
hidróxido de potasio (KOH al 10\%), PAS o mediante cultivo en medio de Sabouraud (16), es importante enfatizar que el diagnóstico definitivo de la $\mathrm{CHC}$ se establece mediante el estudio histopatológico de una biopsia tomada de la lesión, la cual es teñida con PAS u con otro colorante que permita la identificación de la forma filamentosa del hongo (11-15).

El tratamiento de todas las formas de candidiasis oral se basa en el control y corrección de factores locales o sistémicos que favorecen el desarrollo de la candida y simultáneamente administrar terapia antifúngica. Se puede usar polienos tópicos como la nistatina y anfotericina o agentes azoles como clotrimazol, miconazol, ketoconazol, fluconazol o itraconazol (8). En los casos de CHC además de la medicación antifúngica se debe indicar procedimientos mecánicos que permitan desprender al hongo que se encuentra fuertemente adherido a la superficie de la mucosa.

El propósito de este trabajo es reportar el caso de un paciente adulto inmunocompetente diagnosticado con candidiasis hiperplásica crónica del dorso de la lengua. Se presentan y analizan las características clínicas, la probable etiología y el tratamiento realizado. Asimismo, se discuten los cambios histopatológicos en relación con el fenómeno de proliferación y diferenciación celular del epitelio oral.

\section{REPORTE DEL CASO}

Paciente masculino de 54 años de edad, carpintero de profesión, natural de Cerro de Pasco, radicado en Lima desde hace muchos años, sin antecedentes de enfermedades sistémicas, ausencia de hábitos de fumar y abuso de alcohol. Acude al Servicio de Medicina y Cirugía Oral y Maxilofacial de la Facultad de Estomatología de la Universidad Peruana Cayetano Heredia por presentar ardor en el dorso de la lengua desde hace 10 años, menciona que, por indicación profesional, recientemente había tomado una cápsula diaria de fluconazol durante 4 semanas, sin resultados positivos.

La historia dental reveló que había perdido varios dientes superiores por enfermedad periodontal, luego
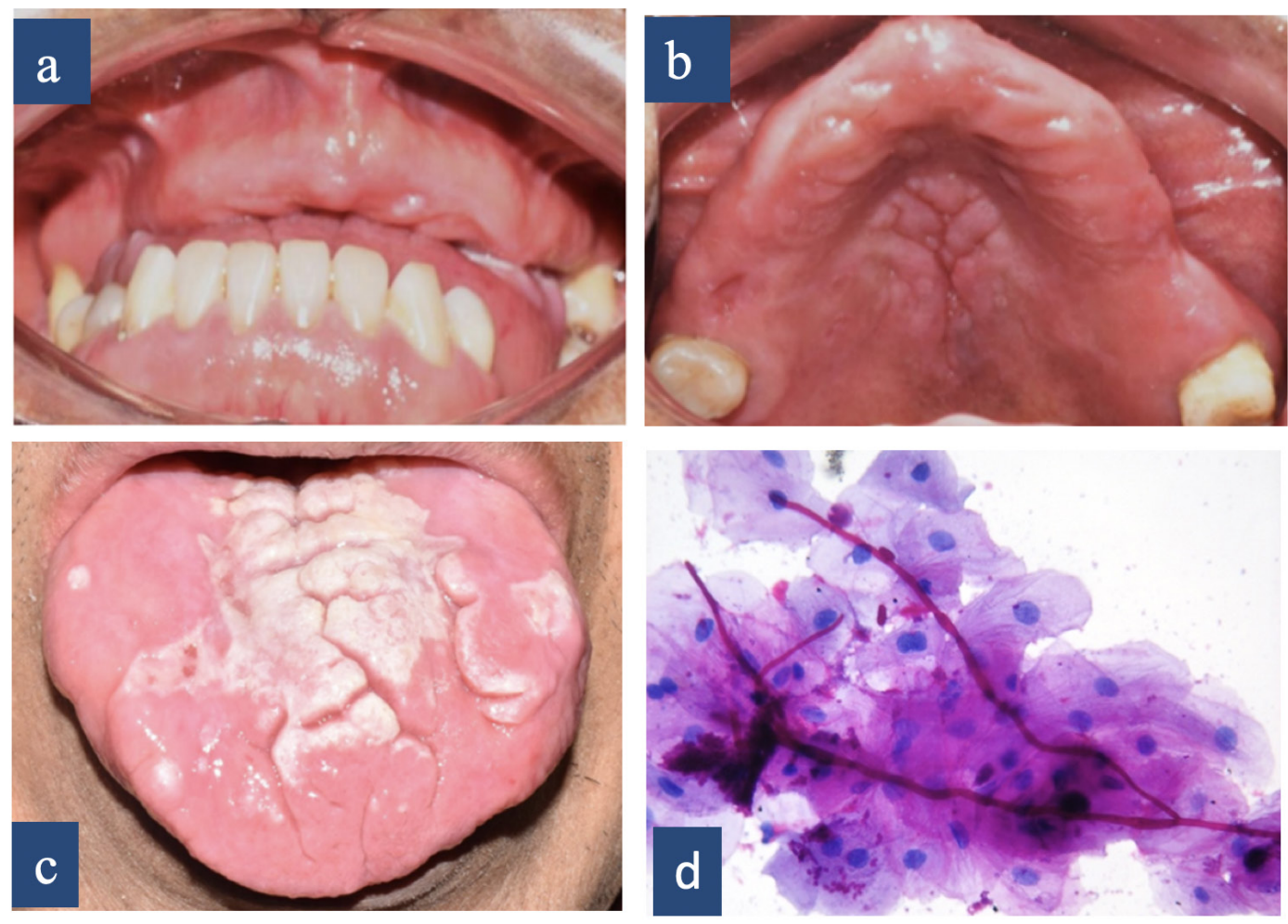

Figura 1. a) Edentulismo parcial superior e inferior y gingivitis marginal. b) Hiperplasia papilar inflamatoria en paladar duro. c) Placa blanca con fisuras que corresponden a CHC. d) Frotis con PAS que muestra hifas y blastosporas de candida y células epiteliales descamadas conteniendo bacterias en su citoplasma $40 \mathrm{X}$. 
usó una prótesis parcial superior cuyos retenedores metálicos le rosaban constantemente la lengua provocándole molestias y heridas cortantes, razón por lo que eliminó estos aditamentos. Actualmente siente que la prótesis parcial superior no está estable.

El examen ectoscópico reveló un paciente en buen estado general. En el examen intraoral se encontró edentulismo parcial superior e inferior, gingivitis marginal crónica en los dientes inferiores asociado a la presencia de placa bacteriana (Fig. 1a). En el paladar duro se detectó una lesión compatible con hiperplasia papilar inflamatoria (Fig. 1b). El flujo salival era normal y portaba una prótesis parcial superior de acrílico en mal estado.

En la zona central del dorso de la lengua se apreciaba una placa amplia, de color blanco, con fisuras que surgían de la línea media, dividiéndola en varias protuberancias (Fig. 1c). En las zonas laterales existían pequeñas pápulas blanquecinas. El resto del dorso lingual presentaba atrofia de papilas filiformes. Ninguna área blanca se desprendía al ser raspada con baja lengua.

Sobre la base de las características clínicas se plantearon los diagnósticos de leucoplasia homogénea y candidiasis hiperplásica crónica. Para establecer el diagnóstico definitivo se realizó un frotis y se tomó una biopsia de una protuberancia blanquecina. El frotis teñido con PAS demostró la presencia de hifas y blastosporas de candida (Fig. 1d).

El examen histopatológico mostraba marcado engrosamiento del epitelio, por incremento del número de las capas superficiales, las células mostraban vacuolización y no existían células queratinizadas ni paraqueratinizadas (Figs. 2a y 2b). Se distinguía marcada hiperplasia pseudoepiteliomatosa, las crestas epiteliales eran delgadas y profundas con hipercelularidad (Figs. 2b y 2c). En las capas basal y
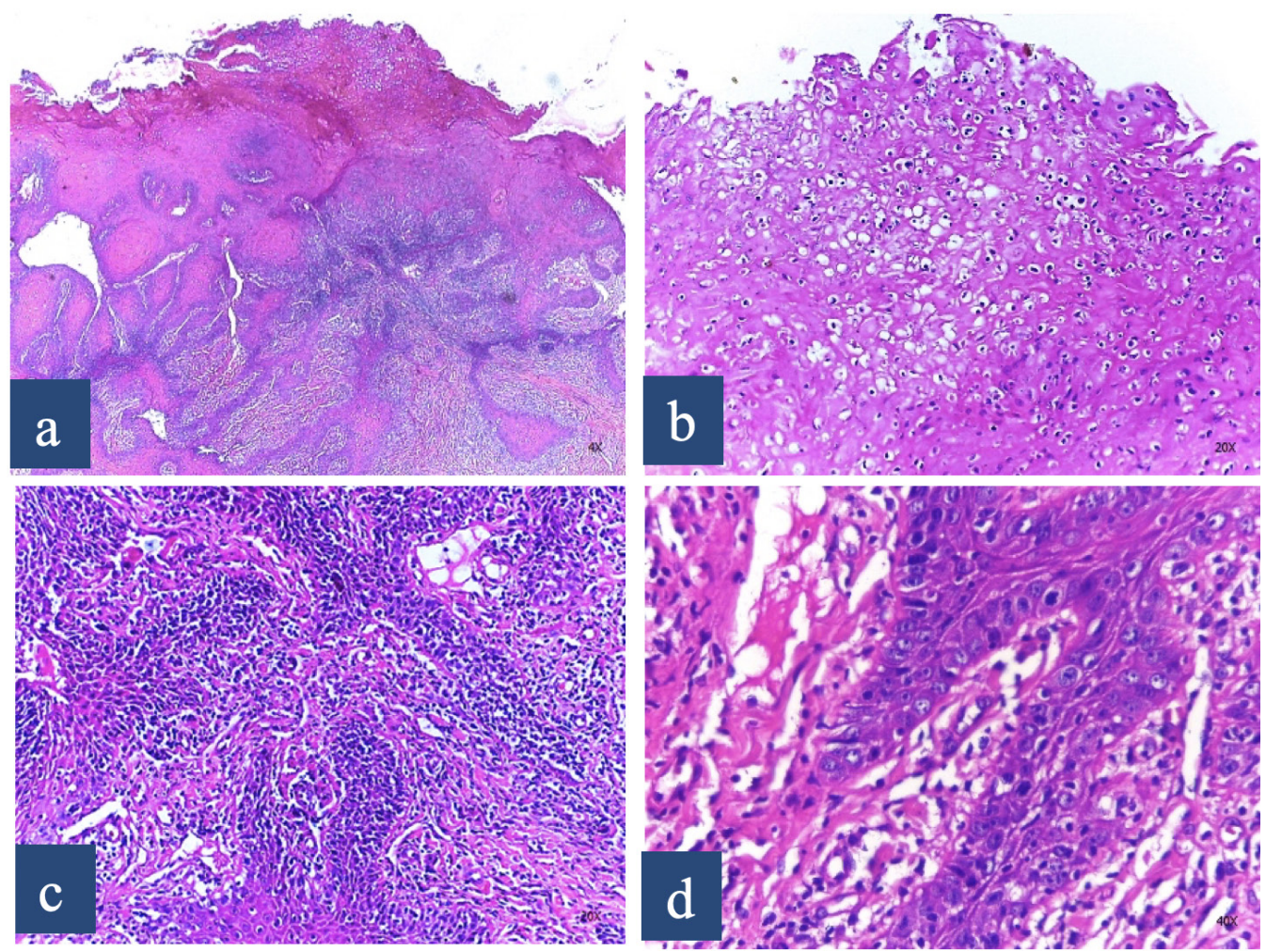

Figura 2. a) Epitelio engrosado donde se observa incremento de las capas superficiales e hiperplasia pseudoepiteliomatosa $H \& E / 4 X$. b) Abundante cantidad de células epiteliales vacuoladas en la superficie epitelial, no se observa signos de queratinización, H\&E/20X. c) Marcada hiperplasia pseudoepiteliomatosa cuyas células muestran hipercromatismo nuclear. H\&E/ 20X. d) Capa basal y parabasal con células con núcleos grandes hipercromáticos y escasas mitosis. H\&E 40X. 

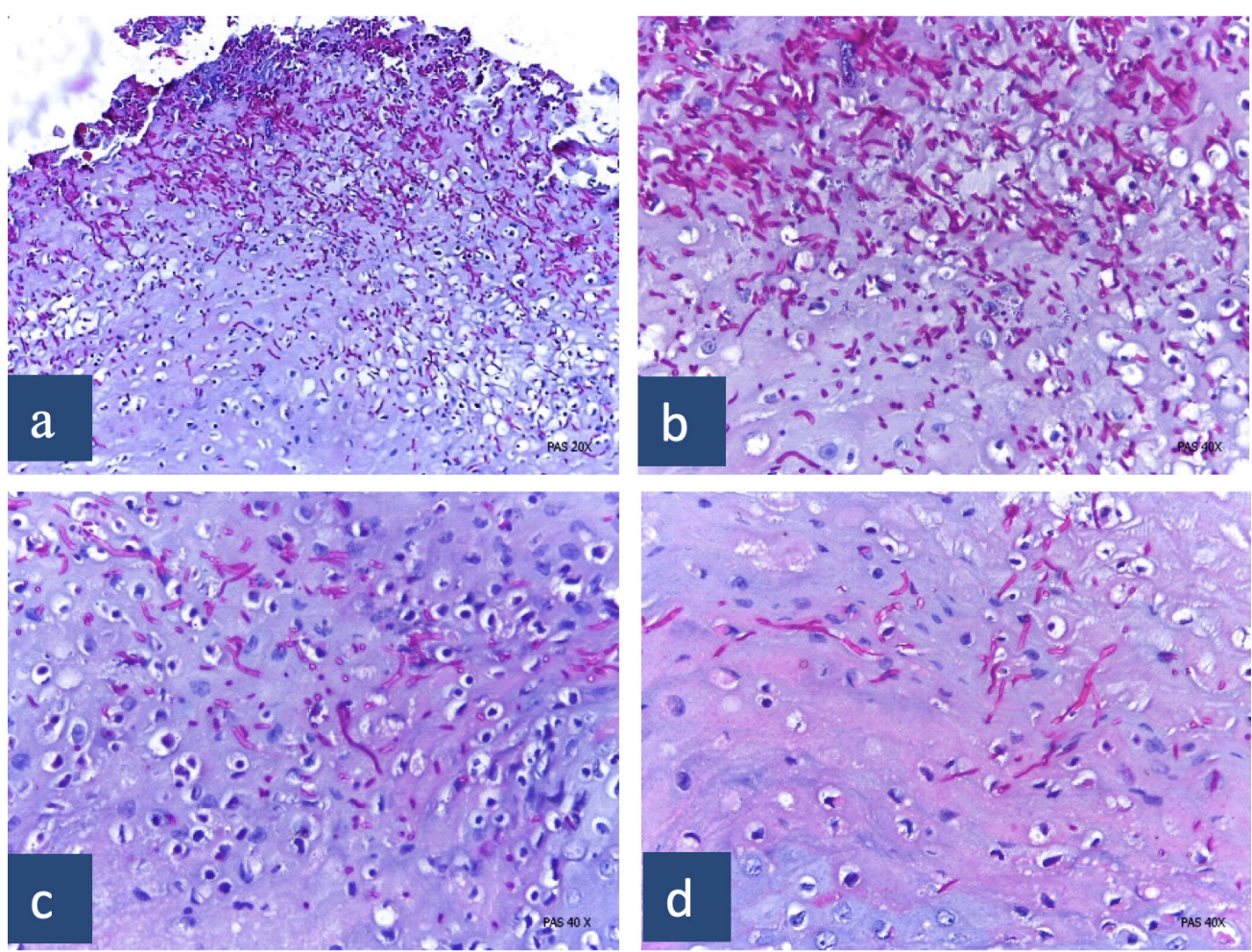

Figura 3. Cortes teñidos con PAS: muestran hifas y blastosporas de candida localizadas en la superficie del epitelio e invadiendo los estratos subyacentes. La mayor concentración del hongo está en el tercio superior del epitelio ( $\mathrm{a}$ y b). En el tercio medio e inferior existe menor cantidad de hifas y blastosporas (c y d).

parabasal se observaban células con hipercromatismo nuclear y algunas mitosis anormales (Fig. 2d). La lámina propia presentaba severo infiltrado inflamatorio linfoplasmocitario.

Los cortes histológicos teñidos con PAS demostraron la presencia de hifas y blastosporas de candida en los diferentes estratos del epitelio. La concentración del hongo fue mayor en el tercio superior, escaso en el tercio medio y mínima en el tercio inferior del epitelio (Figs. 3a, b, c y d). No se observaron hifas en la capa basal ni parabasal. Los hallazgos histopatológicos establecieron el diagnóstico de $\mathrm{CHC}$.

Como tratamiento se indicó una dosis interdiaria de fluconazol de $150 \mathrm{mg}$. por 2 semanas, acompañado de raspado de la lesión con un cepillo dental para niños y enjuagues con clorhexidina al $0,12 \% 3$ veces al día. También se mejoró la higiene dental y se recomendó cambiar la prótesis parcial superior. Al cabo de 14 días los síntomas habían desaparecido y la placa blanca estaba en regresión (Fig. 4). Para

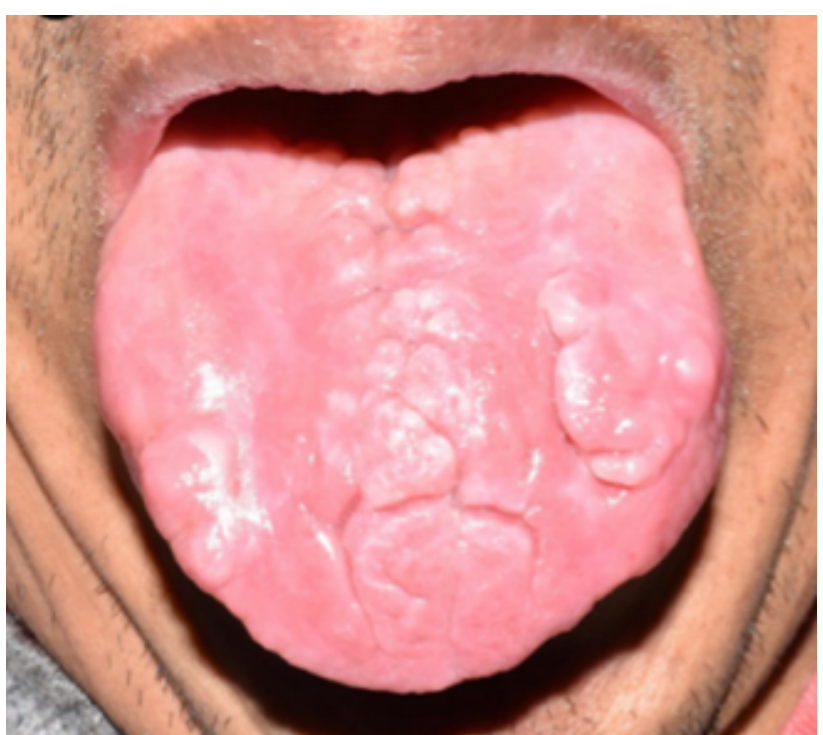

Figura 4. Control a los 14 días del tratamiento: desaparición gradual de la placa blanca y de las fisuras.

completar el tratamiento se prescribió colutorios de nistatina después del desayuno, almuerzo y antes de dormir hasta la desaparición de la lesión blanca. 


\section{DISCUSIÓN}

La candidiasis es la infección micótica más frecuente de la cavidad oral. La candida se considera un comensal y se ha reportado en el $60 \%$ de las bocas de la población sana (2). De las ciento cincuenta especies aislada de la cavidad oral el $80 \%$ corresponde a Candida albicans, en algunos casos puede encontrarse en combinación con C. glabrata o C. tropicalis u otra especie dependiendo del estado inmunológico del individuo (16).

Existen diferentes cuadros clínicos de candidiasis oral los cuales están íntimamente relacionados con el estado sistémico del paciente y con alteraciones del medio ambiente oral que por diversas causas puede ocurrir en la persona. El tratamiento depende del tipo de candidiasis diagnosticada.

La candidiasis hiperplásica crónica representa el $5 \%$ de todas las formas de candidiasis oral, por lo tanto, se considera que es la forma menos frecuente (13). Esta forma de candidiasis presenta dos rasgos sobresalientes: 1. No está asociada a enfermedad sistémica y 2. Se presenta como una placa blanca fuertemente adherida a la superficie de la mucosa que no se desprende al ser raspada o friccionada. Por esta última característica ha sido referida en la literatura como leucoplasia por candida (8), por lo tanto, puede ser confundida con la lesión potencialmente maligna denominada leucoplasia la cual está asociada al desarrollo del carcinoma escamoso de la mucosa oral. En la etiopatogenia de la CHC se considera el hábito de fumar, el trauma y la irritación crónica, de allí que se presente mayormente en zonas que están sometidas a constantes fricciones. El caso que reportamos se desarrolló en el centro del dorso de la lengua, una ubicación muy rara. Su presencia en esta zona podría explicarse porque el paciente manifestó qué durante mucho tiempo, quizás años, había usado una prótesis removible cuyos retenedores metálicos le rosaban el dorso de la lengua produciéndole molestias y leve sangrado, convirtiéndolos en un factor irritativo crónico.

Los cambios histológicos identificados en este estudio, demuestran que el hongo invade los diferentes estratos del epitelio, induciendo alteraciones en el fenómeno de proliferación y diferenciación celular de este tejido, ya que en la superficie no se encontraron células ortoqueratinizadas ni paraqueratinizadas sino células vacuoladas con núcleos grandes (Fig. 2b), por otro lado, las crestas epiteliales mostraban hiperplasia pseudoepiteliomatosa con hipercelularidad y en las capas basal y parabasal se distinguían células con núcleos grandes, hipercromáticos y algunas mitosis atípicas (Figs. 2c y 2d).

La mayor concentración de hifas y blastosporas se encontraron en el tercio superior y su cantidad disminuía en dirección hacia las capas más inferiores del epitelio (Figs. 3a, b, c y d). La presencia del hongo dentro de este tejido sugiere una estrecha relación con las alteraciones en el fenómeno de proliferación y diferenciación celular señaladas. Estos cambios considerados displásicos por algunos autores (11), no se han definido si representan alteraciones con potencial maligno o corresponden a un fenómeno reactivo asociado a la presencia del hongo.

El tratamiento propuesto se fundamentó en el reconocimiento que, en la $\mathrm{CHC}$, la candida forma conjuntamente con las bacterias de la boca una biopelícula o biofilm (5) que se adhiere fuertemente a la superficie de la mucosa y que requiere ser eliminada. Por ello, además de prescribir medicación antifúngica se indicó raspar la placa blanca con un cepillo dental. Los resultados positivos del tratamiento fueron evidentes (Fig.4).

Se concluye que la $\mathrm{CHC}$ es una entidad de gran importancia debido a que clínicamente se parece a la leucoplasia asociada al cáncer oral. $\mathrm{Su}$ comportamiento biológico es muy particular. Para su diagnóstico es indispensable la toma de una biopsia cuyo estudio histológico permita la identificación de hifas y blastosporas de candida invadiendo el epitelio, así como descartar cambios displásicos con potencial de transformación maligna. El tratamiento se basa en la corrección de los factores identificados en la etiología, la eliminación mecánica de la biopelícula micótica y la prescripción de antifúngicos.

\section{Correspondencia:}

Sandro Lévano Loayza

Correo electrónico: sandro.levano.1@gmail.com

\section{REFERENCIAS BIBLIOGRÁFICAS}

1. Correa-Alvarez J, Castro-Martínez S, Coy J. Estado de la moniliasis del cacao causada por Moniliophthora roreri en Colombia. Acta Agron. 2014; 63 (4): 388-99.

2. Martin MV, Lamb DJ. Frequency of Candida albicans 
serotypes in patients with denture-induced stomatitis and in normal denture wearers. J Clin Pathol. 1982; 35(8):888-91.

3. Aguirre-Urízar JM. Oral Candidiasis. Rev Iberoam Micol. 2002; 19:17-21.

4. Scully C, el-Kabir, Samaranayake LP. Candida and oral candidosis: a review. Crit Rev Oral Biol Med. 1994; 5:125-57.

5. Rautemaa R, Ramage G. Oral candidosis - Clinical challenges of a biofilm disease. Crit Rev in Microbiol. 2011; 37(4):328-36.

6. Holmstrup P, Axell T. Classification and clinical manifestations of oral yeast infections. Acta Odontol Scand. 1990; 48:57-9.

7. Neville BW, Damm DD, Allen CM, Bouquot JE. Oral and Maxillofacial Pathology. 3rd ed. Philadelphia: W.B. Sauders; 2009.p. 218-21.

8. Patil S, Rao RS, Majumdar B, Anil S. Clinical Appearance of Oral Candida infection and therapeutic strategies. Front Microbiol. 2015: 6:1391. doi: 10.3389/ fmicb.2015.01391

9. Farah CS, Lynch N, McCullough MJ. Oral fungal infections: an update for the general practitioner. Australian Dent J. 2010; 55(1suppl): 48-54.

10. Teria H, Shimahara M. Atrophic tongue associated with candida. J Oral Pathol Med. 2005; 34(7): 397-400.
11. Sitheeque MAM, Samaranayake LP. Chronic hyperplastic candidosis/candidiasis (candida leukoplakia).Clin Rev Oral Biol Med. 2003; 14: 25367.

12. Gracia MTP, Fernández CMH, Cebrian BM, García BS. Chronic Hyperplastic Candidiasis Oral fungal infections: an update for the general practitoner. Australian Dent J. 2010; 55: (1Suppl): 48-54.

13. Gonzales-García RJ, Sastre-Perez MF, Muñoz-Guerra $\mathrm{L}$, et al. Candidiasis hperplásica crónica de la mucosa oral. Rev Esp Cir Oral y Maxilofac. 2006; 28: 191-94.

14. Gracia MTP, Fernández CMH, Cebrian BM, García BS Chronic Hyperplastic Candidiasis of the oral mucosa: Case Report. J Clin Stud Med Case Rep. 2014;1:1-3.

15. Hellstein JW, Marek CL. Candidiasis: Red and White Manifestations in the Oral Cavity. Head and Neck Pathol. 2019; 13:25-32.

16. Coronado-Castellote L, Jiménez-Soriano Y. Clinical and microbiological diagnosis of candidiasis. J Clin Exp Dent. 2013;5(5):e279-86.

Recibido : 27-08-2020

Aceptado : 26-08-2021 